\title{
Association of centre volume and in-hospital mortality in heart failure hospitalisations
}

\author{
Shivank Madan, Daniel Sims, Omar Saeed, Snehal R Patel, Jooyoung J Shin, \\ Ulrich P Jorde
}

- Additional material is published online only. To view please visit the journal online (http://dx.doi.org/10.1136/ postgradmedj-2016-134697).

Division of Cardiology, Albert Einstein College of MedicineMontefiore Medical Center, New York City, New York, USA

\section{Correspondence to} Dr Shivank Madan, Cardiology Fellow, Albert Einstein College of Medicine/Montefiore Medical Center, 3400 Bainbridge

Avenue, Medical Arts Pavillion7th floor, Bronx, NY 10467,

USA; smadan@montefiore.org

Received 27 November 2016 Revised 25 January 2017 Accepted 13 February 2017 Published Online First 2 March 2017

\section{CrossMark}

To cite: Madan S, Sims D,

Saeed 0 , et al.

Postgrad Med J

2017;93:534-540.

\section{ABSTRACT \\ Background Centre volume is an important} determinant of outcomes in patients requiring complex medical treatments or surgical procedures. Heart failure hospitalisation (HFH) has become an increasingly complex and resource intensive clinical event. We evaluated the effect of centre volume on mortality and costs in patients with $\mathrm{HFH}$.

Methods This was a retrospective registry-based analysis of adult patients discharged with a primary diagnosis of HF from hospitals across New York (NY) State over a 5-year period, between January 2009 and December 2013, using the Statewide Planning and Research Cooperative System inpatient discharge files. The primary outcome of interest was in-hospital mortality. All patients were followed from the day of admission to either in-hospital death or discharge alive. Results 300972 HFHs from 198 facilities across NY State were included. Five-year centre volume was associated with a decrease in in-hospital mortality in unadjusted ( $\mathrm{HR}=0.872,95 \% \mathrm{Cl} 0.863$ to 0.881 , $\mathrm{p}<0.001)$ and adjusted Cox models $(H R=0.869,95 \%$ $\mathrm{Cl} 0.859$ to $0.879, \mathrm{p}<0.001)$. After dividing the overall cohort into three groups based on 5-year centre volume, groups with medium and high volume centres had lower in-hospital mortality when compared with the group with low volume centres. The results were consistent in various subgroup analyses. Furthermore, hospitals in the higher centre volume groups had increased HFH costs across different severity of illness categories and involved increased use of cardiac procedures.

Conclusions Higher centre volume was associated with lower HFH mortality but increased HFH costs and increased cardiac procedures in a cohort of Medicare and non-Medicare beneficiaries.

\section{INTRODUCTION}

Heart failure (HF) is the most common cause of hospitalisation in patients over the age of 65 years in the USA. ${ }^{1}$ HF costs the US healthcare system about US $\$ 32$ billion every year, ${ }^{2}>50 \%$ of which is incurred during $\mathrm{HF}$ hospitalisations ( $\mathrm{HFH}) .{ }^{3} \mathrm{HFH}$ is also a major clinical event with 1 year mortality rates following HFH approaching $29.6 \%{ }^{4}$ Hence, there is an increased interest in identifying standards of care, and improve quality of healthcare services being delivered to patients with HFH. Furthermore, as more therapeutic options have become available and as patients with HF have an increased burden of comorbidities, caring for patients with HF has become increasingly complex and resource intensive. ${ }^{5}$

Centre volume is a major determinant of outcomes in heart transplants, ${ }^{6}$ coronary interventions, ${ }^{7}$ burn injuries ${ }^{8}$ and trauma patients. ${ }^{9}$ While the association between higher centre volumes and better health outcomes is strongest for surgical procedures, studies have shown a strong relationship between higher centre volumes and lower in-hospital mortality in other complex diseases like patients with HIV/AIDS ${ }^{10}$ and in patients undergoing chemotherapy. ${ }^{11}$ The objective of our study was to evaluate the relationship between centre volume, $\mathrm{HFH}$ mortality and HFH costs in both Medicare and non-Medicare patients with HF.

\section{METHODS}

\section{Study design}

In this retrospective registry-based analysis, we identified patients with a primary discharge diagnosis of HF in New York (NY) State from 1 January 2009 to 31 December 2013.

\section{Registries}

Patients included in this study were identified from the Statewide Planning and Research Cooperative System (SPARCS) inpatient discharge files provided by the New York State Department of Health $(\mathrm{NYSDOH})$ using the Clinical Classification Software (CCS) tool developed at the Agency for Healthcare Research and Quality. SPARCS database contains information about patient discharges from non-federal acute care hospitals in NY State and all facilities are required to submit this data on a monthly basis. SPARCS contains data on patient characteristics, diagnosis and treatments for each in-hospital stay. Since these data provided by NY State is de-identified, no separate informed consent was required. The study was approved by the Institutional Review Board at Albert Einstein College of Medicine/Montefiore Medical Center. Details regarding the SPARCS/NYSDOH data registry and CCS tool are provided in the online supplementary appendix.

\section{Study population}

Patients were eligible to be included in the study if they were discharged with a primary diagnosis of HF. CCS code 108 was used to identify a HF discharge. CCS code 108 contains the following International Classification of Diseases-ninth revision codes: 428.0 (congestive HF unspecified), 428.1 (left HF), 428.20 (unspecified systolic HF), 428.21 (acute systolic HF), 428.22 (chronic systolic HF), 428.23 (acute on chronic systolic HF), 428.30 (unspecified diastolic HF), 428.31 (acute diastolic HF), 428.32 (chronic diastolic HF), 428.33 (acute on chronic diastolic HF), 428.40 (unspecified combined systolic and diastolic HF), 428.41 (acute unspecified combined systolic and diastolic HF), 
428.42 (chronic unspecified combined systolic and diastolic HF), 428.43 (acute on chronic unspecified combined systolic and diastolic HF), 428.9 (HF unspecified) and 398.91 (rheumatic HF).

Paediatric HFH (<18 years) or HFH with incomplete information were excluded (see online supplementary figure S1). Since, the unit of record for this database was HFH, all analysis were done using $\mathrm{HFH}$ as a unit, rather than each individual patient.

\section{Study variables}

The following information was retrieved from the hospital inpatient discharge (SPARCS de-identified) files for each hospital stay with a diagnosis of HF: health service area, hospital facility identification number (ID), age category in years $(0-17,18-29$, $30-49,50-69$ and 70 or older), gender, race and ethnicity, length of hospital stay, type of admission, patient disposition, discharge year, all patient refined diagnosis related groups (APR-DRGs) and payer status. Developed as a modification to DRGs, the APR-DRGs contain information on APR-risk of mortality (APR-ROM) and APR-severity of illness (APR-SOI). The APR-ROM refers to 'likelihood of dying' and APR-SOI refers to the 'extent of physiologic decompensation or organ system loss of function'. Both APR-SOI and APR-ROM are classified into four subcategories as minor, moderate, major or extreme and help to determine disease severity and case mix complexity. APR-DRGs have been clinically validated and used for case-mix index in previous studies. ${ }^{12}{ }^{13}$ A patient was considered to be admitted from an emergency department (ED) based on the ED ancillary revenue codes ( $045 \mathrm{X}$ series) submitted by the hospital. 'Centre volume' of a facility was defined as the total number of HFH in that facility during the 5-year study period. All the hospitals were analysed using the 'facility ID' instead of 'facility name' as this ensured change of facility name or change of affiliation did not affect our analysis, since 'facility ID' remains constant in SPARCS.

\section{Outcomes}

The primary outcome of interest was in-hospital mortality coded at the time of discharge.

\section{Follow up}

Patients were followed for up to 120 days from the date of admission during which $99.95 \%$ were either discharged alive or died in the hospital.

\section{Statistical analysis}

Baseline characteristics were described as frequencies (percentage) for categorical variables, mean \pm SD for continuous variables with a normal distribution, and median (first and third quartiles: Q1-Q3) for continuous variables with a non-normal distribution; $\chi^{2}$ test was used for categorical variables, analysis of variance for continuous variables with a normal distribution and Wilcoxon rank test for continuous variables with a nonnormal distribution.

To assess the association between HFH mortality and centre volume, we conducted a survival analysis using Cox proportional hazards regression with the length of hospital stay as the time scale (to adjust for temporal differences in mortality) and in-hospital death as an outcome. First, we evaluated the effect of centre volume on in-hospital mortality, using centre volume as a continuous variable in both unadjusted and adjusted Cox models. Next, we divided the overall cohort of HF discharges in the NY State over 5-year study period into three groups, stratified on the number of HFH per facility over the 5-year period. This ensured that each group had about one-third of the
$\mathrm{HFH}$ and there was a graded increase in the 5 -year HFH from group one (low volume) to group three (high volume). These three groups were compared for baseline characteristics, in-hospital mortality and HFH costs.

Finally, we also created a multivariable Cox proportional hazards model using a backward stepwise selection method to analyse the independent effects of each variable on in-hospital mortality with a stay significance levels of $<0.001$. The variables included in the analysis were age $(<70$ or $\geq 70$ years), gender, race (blacks vs others), ethnicity (Hispanic vs others), APR-SOI (minor/moderate vs major/extreme), APR-ROM (minor/moderate vs major/extreme), primary insurance (Medicare/Medicaid/ other federal programmes vs other insurance or self-pay), admission through ED (yes or no) and centre volume. Two tailed $p$ values were used and $p<0.001$ was considered to be statistically significant. All statistical analysis was conducted with STATA V.13 software.

\section{RESULTS}

\section{Study population and baseline characteristics}

From $301751 \mathrm{HFH}$ in SPARCS/NYSDOH database during the study period (January 2009 to December 2013), 300972 (99.74\%) met all study criteria and were included in the current analysis. We found that there was a small reduction in the total number of yearly HFH, from 66019 in 2009 to 57402 in 2013, but the burden of HFH still remains high (see online supplementary figure $\mathrm{S} 1$ ).

Baseline characteristics of the overall cohort are shown in table 1. Thirty-four per cent of the overall population was below 70 years. The number of HFH with male and female patients was approximately equal; $21.4 \%$ of patients were blacks and $10.18 \%$ identified themselves as having Hispanic origin; $53.8 \%$ of the HFH had APR-SOI categorised as 'major' or 'extreme' and $45.7 \%$ of the HFH had APR-ROM as 'major' or 'extreme'. Based on the ED revenue codes, patients were admitted through the ED in about $83 \%$ of the HFH. Medicare, Medicaid or other federal programmes together accounted for $74.28 \%$ of the primary insurance payers and $22.2 \%$ were from other private insurers (table 1).

Baseline characteristics of HFH according to the three centre volume groups are shown in table 2 . On comparing different centre volume groups, as shown in table 3, there were 132, 44 and 22 hospitals in groups 1 (low volume), 2 (medium volume) and 3 (high volume), respectively. All the hospitals in the 'low volume group' had a 5 -year centre volume of $\leq 1718 \mathrm{HFH}$ while hospitals in the 'high volume group' had a 5 -year centre volume of $\geq 3090 \mathrm{HFH}$ (table 3).

\section{Centre volume, in-hospital mortality, costs and procedures}

There were 12540 in-hospital deaths during the study period. The crude in-hospital mortality rate was $4.16 \%$ for the overall cohort. When analysed as a continuous variable, higher centre volume (measured in thousands) was associated with a lower in-hospital mortality in unadjusted Cox model ( $\mathrm{HR}=0.872$, $95 \%$ CI 0.863 to $0.881, \mathrm{p}<0.001$ ) (table 1 ). This relationship persisted in the adjusted Cox model as well ( $\mathrm{HR}=0.869,95 \%$ CI 0.859 to $0.879, \mathrm{p}<0.001$ ) after adjusting for age category, gender, race, ethnicity, APR-SOI, APR-ROM, type of primary insurance and admission through ED.

On comparing in-hospital mortality in the three centre volume groups using Cox models, medium and high centre volume groups had lower in-hospital mortality compared with the low centre volume group. This association remained significant after adjusting for differences in the baseline characteristics 
Table 1 Baseline characteristics and HR for in-hospital mortality in the overall cohort

\begin{tabular}{|c|c|c|c|}
\hline Baseline characteristics & Overall cohort $(n=300972)$ & HRs $(95 \% \mathrm{Cl})$ & p Value \\
\hline \multicolumn{4}{|l|}{ Age category (years) } \\
\hline$<70$ & $102510(34.06 \%)$ & 2.855 (2.721 to 2.995$)$ & $<0.001$ \\
\hline$\geq 70$ & $198462(65.94 \%)$ & & \\
\hline Gender (females) & $150614(50.04 \%)$ & $1.079(1.042$ to 1.118$)$ & $<0.001$ \\
\hline Race (black vs others) & $64367(21.39 \%)$ & 0.515 (0.489 to 0.544$)$ & $<0.001$ \\
\hline Ethnicity (Hispanic vs others) & $30633(10.18 \%)$ & 0.751 (0.703 to 0.803$)$ & $<0.001$ \\
\hline \multicolumn{4}{|l|}{ APR-severity of illness } \\
\hline Minor/moderate & $139158(46.24 \%)$ & & \\
\hline Major/extreme & $161814(53.76 \%)$ & 2.393 (2.267 to 2.526$)$ & $<0.001$ \\
\hline \multicolumn{4}{|l|}{ APR-risk of mortality } \\
\hline Minor/moderate & $163437(54.30 \%)$ & & \\
\hline Major/extreme & $137535(45.70 \%)$ & 4.037 (3.818 to 4.269$)$ & $<0.001$ \\
\hline \multicolumn{4}{|l|}{ Primary insurance } \\
\hline Medicare/Medicaid/federal programme & $223560(74.28 \%)$ & 1.352 (1.294 to 1.412$)$ & $<0.001$ \\
\hline Other insurance and self-pay & $77412(25.72 \%)$ & & \\
\hline Admission through emergency department (yes $=1, \mathrm{no}=0$ ) & $249722(82.97 \%)$ & $0.816(0.783$ to 0.851$)$ & $<0.001$ \\
\hline Five-year centre volume (in thousands) & $1.181(0.493-2.017)$ & $0.872(0.863$ to 0.881$)$ & $<0.001$ \\
\hline
\end{tabular}

Table 2 Characteristics of HFH according to centre volume groups

\begin{tabular}{|c|c|c|c|c|}
\hline & $\begin{array}{l}\text { Group } 1 \text { (low volume) } \\
(\mathrm{n}=98 \text { 794) }\end{array}$ & $\begin{array}{l}\text { Group } 2 \text { (medium volume) } \\
(n=100813)\end{array}$ & $\begin{array}{l}\text { Group } 3 \text { (high volume) } \\
(n=101365)\end{array}$ & $\begin{array}{l}p \text { Value for difference } \\
\text { between groups }\end{array}$ \\
\hline \multicolumn{5}{|l|}{ Age groups (years) } \\
\hline $18-29$ & 287 (0.29\%) & $365(0.36 \%)$ & $595(0.59 \%)$ & \multirow[t]{4}{*}{$<0.001$} \\
\hline $30-49$ & 4999 (5.06\%) & $5850(5.80 \%)$ & $6654(6.56 \%)$ & \\
\hline $50-69$ & $25405(25.72 \%)$ & $28337(28.11 \%)$ & $30018(29.61 \%)$ & \\
\hline 70 or older & $68103(68.93 \%)$ & $66261(65.73 \%)$ & $64098(63.23 \%)$ & \\
\hline \multicolumn{5}{|l|}{ Gender } \\
\hline Females & $51210(51.84 \%)$ & $51063(50.65 \%)$ & $48341(47.69 \%)$ & \multirow[t]{2}{*}{$<0.001$} \\
\hline Males & $47584(48.16 \%)$ & $49750(49.35 \%)$ & $53024(52.31 \%)$ & \\
\hline \multicolumn{5}{|l|}{ Race } \\
\hline Whites & $69179(70.02 \%)$ & $60590(60.10 \%)$ & 59937 (59.13\%) & \multirow[t]{3}{*}{$<0.001$} \\
\hline Blacks & $16465(16.67 \%)$ & $25223(25.02 \%)$ & $22679(22.37 \%)$ & \\
\hline Others & $13150(13.31 \%)$ & $15000(14.88 \%)$ & $18749(18.50 \%)$ & \\
\hline \multicolumn{5}{|l|}{ Ethnicity } \\
\hline Hispanic & 9462 (9.58\%) & $10265(10.18 \%)$ & $10906(10.76 \%)$ & $<0.001$ \\
\hline \multicolumn{5}{|l|}{ APR-severity of illness } \\
\hline Minor & 7714 (7.81\%) & 8471 (8.40\%) & 7949 (7.84\%) & \multirow[t]{4}{*}{$<0.001$} \\
\hline Moderate & $42146(42.66 \%)$ & $38546(38.24 \%)$ & $34330(33.87 \%)$ & \\
\hline Major & $40760(41.26 \%)$ & 43897 (43.54\%) & $47305(46.67 \%)$ & \\
\hline Extreme & $8172(8.27 \%)$ & 9899 (9.82\%) & $11781(11.62 \%)$ & \\
\hline \multicolumn{5}{|l|}{ APR risk of mortality } \\
\hline Minor & $11467(11.61 \%)$ & $12485(12.38 \%)$ & $10724(10.58 \%)$ & \multirow[t]{4}{*}{$<0.001$} \\
\hline Moderate & $43815(44.35 \%)$ & 42277 (41.94\%) & $42667(42.09 \%)$ & \\
\hline Major & $32855(33.26 \%)$ & $33655(33.38 \%)$ & 34919 (34.45\%) & \\
\hline Extreme & $10655(10.79 \%)$ & $12396(12.30 \%)$ & $13055(12.88 \%)$ & \\
\hline \multicolumn{5}{|l|}{ Primary source of payment } \\
\hline Other Insurance & $17997(18.22 \%)$ & 21991 (21.81\%) & $26859(26.50 \%)$ & \multirow[t]{4}{*}{$<0.001$} \\
\hline Medicare/Medicaid/federal programme & $76836(77.77 \%)$ & $73625(73.03 \%)$ & $73099(72.11 \%)$ & \\
\hline Self-pay & $3912(3.96 \%)$ & $5077(5.04 \%)$ & $1309(1.29 \%)$ & \\
\hline Undetermined & $49(0.05 \%)$ & $120(0.12 \%)$ & $98(0.10 \%)$ & \\
\hline Admission via emergency department & $84878(85.91 \%)$ & $87619(86.91 \%)$ & 77225 (76.19\%) & $<0.001$ \\
\hline
\end{tabular}

\footnotetext{
$\mathrm{HFH}$, heart failure hospitalisation.
} 
Table 3 Centre volume groups and in-hospital mortality

\begin{tabular}{|c|c|c|c|c|c|}
\hline & $\begin{array}{l}\text { Number of } \\
\text { hospitals }\end{array}$ & $\begin{array}{l}\text { 5-year centre volume: } \\
\text { median (Q1-Q3) }\end{array}$ & $\begin{array}{l}\text { LOS in days median } \\
\text { (Q1-Q3) }\end{array}$ & $\begin{array}{l}\text { Unadjusted HR }(95 \% \mathrm{Cl}) \text {, } \\
\text { p value }\end{array}$ & $\begin{array}{l}\text { Adjusted } \mathrm{HR}^{*}(95 \% \mathrm{CI}) \text {, } \\
\text { p value }\end{array}$ \\
\hline Low volume ( $\leq 1718 \mathrm{HFH})$ & 132 & $1085(728-1424)$ & $4(3-7)$ & Reference & Reference \\
\hline Medium volume (1721-3080 HFH) & 44 & $2438(1988-2759)$ & $5(3-8)$ & $0.75(0.72$ to 0.78$), p<0.001$ & $0.74(0.71$ to 0.77$), p<0.001$ \\
\hline High volume ( $\geq 3090 \mathrm{HFH})$ & 22 & $5030(3699-5977)$ & $5(3-8)$ & 0.61 (0.58 to 0.64$), \mathrm{p}<0.001$ & $0.58(0.56$ to 0.61$), p<0.001$ \\
\hline
\end{tabular}

(table 3). Figure 1 shows the Kaplan-Meier estimates of cumulative in-hospital mortality in the three centre volume groups at different lengths of hospital stay.

To check the robustness of our findings, in sub group analysis, we looked at patients admitted via the ED $(n=249722)$ or not $(n=51250)$. This was done to check for any bias that may have occurred due to transfer of patients from one facility to another for advanced management of HF. However, in survival models, higher centre volume was still associated with a lower in-hospital mortality, irrespective of the fact whether the patients were admitted via ED or not (table 4). The results were consistent in other subgroup analyses as well, including in $\mathrm{HFH}$ involving younger ( $<70$ years) and non-Medicare patients (table 4). Finally, we also compared crude mortality rates in the three centre volume groups based on APR-ROM and APR-SOI. There was a significant decrease in crude mortality rates from low to high centre volume in various subgroups as well (see online supplementary table B).

We also analysed the total hospital costs per HFH according to centre volume group and APR-SOI category. There was a significant trend towards increased HFH costs from low volume to higher volume centre groups for HFH with both 'mild or moderate' and 'major or extreme' APR-SOI (table 5). Finally, we also looked at the highest billed cardiac procedures for each $\mathrm{HFH}$ across various centre volumes. As expected, there was a significant trend for increased use of diagnostic cardiac catheterisation, percutaneous transluminal coronary angioplasty and insertion/ removal/replacement of pacemakers or cardioverter defibrillator from low to high centre volumes $(p<0.001$ for trend analysis, table 6).

\section{Association between other variables and in-hospital mortality}

In the multivariate Cox model to identify the independent effect of each variable (listed in table 1), age $\geq 70$ years, APR-SOI, APR-ROM were associated with increased HR for in-hospital mortality, whereas black race, Hispanic ethnicity and admission via ED along with centre volume were found to be significantly associated with reduced HR for in-hospital mortality (see online supplementary table A). More importantly, type of primary insurance and gender did not have any significant effect on in-hospital mortality in the multivariate model.

\section{DISCUSSION}

We examined the association of centre volume and in-hospital mortality in over $300000 \mathrm{HFH}$ over a 5-year period in NY State. Our principal findings are:

a. Centre volume is strongly associated with HFH mortality in a cohort that included Medicare and non-Medicare patients (table 3).

b. Hospitals in the higher centre volume groups had lower HFH mortality but increased HFH costs and increased use

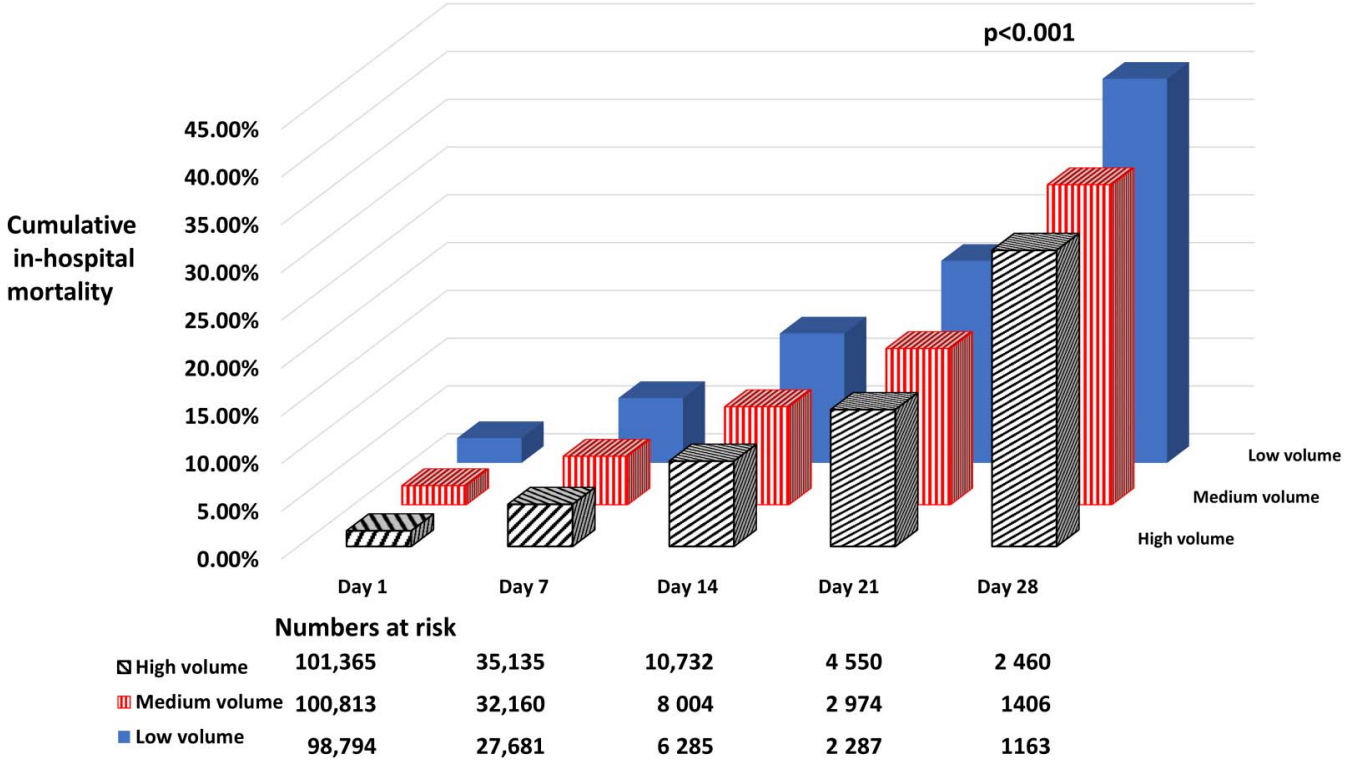

Figure 1 Cumulative in-hospital mortality across different centre volume groups at different lengths of hospital stay. 
Table 4 Five-year centre volume and in-hospital mortality in various subgroups

\begin{tabular}{|c|c|c|c|c|}
\hline & Unadjusted HR $(95 \% \mathrm{CI})$ & $p$ Value & Adjusted HR* $(95 \% \mathrm{CI})$ & $p$ Value \\
\hline \multicolumn{5}{|l|}{ Admitted via emergency department? } \\
\hline Yes $(n=249722)$ & 0.895 (0.884 to 0.906$)$ & $<0.001$ & 0.906 (0.894 to 0.917$)$ & $<0.001$ \\
\hline No $(n=51250)$ & $0.799(0.783$ to 0.815$)$ & $<0.001$ & $0.823(0.806$ to 0.840$)$ & $<0.001$ \\
\hline \multicolumn{5}{|l|}{ Age category } \\
\hline$<70$ years $(n=102510)$ & $0.887(0.865$ to 0.909$)$ & $<0.001$ & $0.862(0.840$ to 0.884$)$ & $<0.001$ \\
\hline$\geq 70$ years $(n=198462)$ & $0.892(0.882$ to 0.903$)$ & $<0.001$ & 0.881 (0.870 to 0.891$)$ & $<0.001$ \\
\hline \multicolumn{5}{|l|}{ Gender } \\
\hline Females ( $n=150614)$ & 0.874 (0.861 to 0.887$)$ & $<0.001$ & $0.883(0.870$ to 0.897$)$ & $<0.001$ \\
\hline Males $(n=150358)$ & 0.870 (0.857 to 0.883$)$ & $<0.001$ & 0.871 (0.858 to 0884$)$ & $<0.001$ \\
\hline \multicolumn{5}{|l|}{ Race } \\
\hline Blacks ( $n=64367$ ) & $0.898(0.872$ to 0.926$)$ & $<0.001$ & 0.900 (0.873 to 0.928$)$ & $<0.001$ \\
\hline Others $(n=236605)$ & 0.975 (0.866 to 0.885$)$ & $<0.001$ & $0.876(0.866$ to 0.885$)$ & $<0.001$ \\
\hline \multicolumn{5}{|l|}{ Ethnicity } \\
\hline Hispanics $(n=30633)$ & 0.875 (0.846 to 0.906$)$ & $<0.001$ & $0.888(0.858$ to 0.920$)$ & $<0.001$ \\
\hline Others $(n=270339$ ) & $0.873(0.863$ to 0.883$)$ & $<0.001$ & 0.876 (0.866 to 0.886$)$ & $<0.001$ \\
\hline \multicolumn{5}{|l|}{ APR-severity of illness } \\
\hline Minor/moderate $(n=139$ 158) & 0.771 (0.744 to 0.798$)$ & $<0.001$ & 0.796 (0.769 to 0.822$)$ & $<0.001$ \\
\hline Major/extreme $(n=161814)$ & $0.872(0.862$ to 0.881$)$ & $<0.001$ & 0.891 (0.881 to 0.901$)$ & $<0.001$ \\
\hline \multicolumn{5}{|l|}{ APR-risk of mortality } \\
\hline Minor/moderate ( $n=163$ 437) & $0.741(0.715$ to 0.768$)$ & $<0.001$ & 0.774 (0.748 to 0.802$)$ & $<0.001$ \\
\hline Major/extreme $(n=137535)$ & 0.877 (0.867 to 0.886$)$ & $<0.001$ & 0.891 (0.881 to 0.901$)$ & $<0.001$ \\
\hline \multicolumn{5}{|l|}{ Primary insurance } \\
\hline Medicare/Medicaid/other federal programme $(n=223560)$ & 0.845 (0.826 to 0.865$)$ & $<0.001$ & $0.883(0.872$ to 0.894$)$ & $<0.001$ \\
\hline Other insurance $(n=77412)$ & $0.884(0.873$ to 0.894$)$ & $<0.001$ & 0.854 (0.834 to 0.874$)$ & $<0.001$ \\
\hline
\end{tabular}

of cardiac procedures (see online supplementary table B, tables 5 and 6).

c. While the relative risk reduction in $\mathrm{HFH}$ mortality was modest for the sickest group of patients (HFH with both APR-SOI and APR-ROM 'major or extreme'), the increase in costs was much more significant (see online supplementary table $\mathrm{B}$ and table 5).

Over the past decade, there has been growing evidence supporting the 'centre volume and outcomes' effect, such that patients requiring surgical procedures or complex medical treatments have better outcomes at centres with higher patient volumes. ${ }^{67}$ In our study, we found a similar effect, that is, there was a reduction in 'in-hospital mortality' with increase in centre volume in $\mathrm{HFH}$ across NY State during the 5-year study period (2009-2013). This relationship persisted in various subgroup analyses as well (table 4). While, a previous study about centre volume and HF outcomes included only older Medicare beneficiaries, ${ }^{14}$ the current analysis of centre volume, HFH mortality and HFH costs includes both Medicare and non-Medicare (Medicaid, other federal programme and private insurance) patients.
Although in our analysis, we cannot point to an exact reason for the observed reduction in 'in-hospital mortality' with an increase in 'centre volume' in patients with HF, this could be due to a variety of reasons. HFH is a complex diagnosis requiring a team-based approach involving primary care physicians, hospitalists, cardiologists, HF specialists, nurses, pharmacists and nutritionists who are trained and experienced in taking care of patients with HF. It is possible that higher volume centres have better resources at their disposal to care for patients with HF. Higher volume centres may have a better physical infrastructure, better adherence to treatment guidelines and understanding of the care required by patients with HF. Studies in patients undergoing cardiac surgeries have shown that higher performing centres do not necessarily have a lower rate of complications, but are likely to be better at managing complications once they occur. ${ }^{15}$ patients with $\mathrm{HF}$ are at high risk for complications like sudden cardiac death, progressive pump failure, acute kidney injury, liver failure, intestinal ischaemia and electrolyte abnormalities ${ }^{16-18}$ and it is possible that centres with high volumes may have more experience in taking care of these complications.

Table 5 HFH costs in different centre volume groups based on APR-severity of illness categories

\begin{tabular}{llcc}
\hline APR-severity of illness & Low volume & Medium volume & High volume \\
\hline Mild or moderate & US\$4938 (3253-7692) & US\$5652 (3672-8715) & US\$5686 (3507-9959) \\
Major or extreme & US\$8682 (5323-15 447) & US\$10 869 (6418-20 115) & US\$13 948 (7154-31 873) \\
\hline
\end{tabular}

APR, all patient refined; $H F H$, heart failure hospitalisation. 
Table 6 Cardiac procedures across different centre volume groups

\begin{tabular}{|c|c|c|c|c|}
\hline & Low volume & Medium volume & High volume & p Value \\
\hline Percutaneous transluminal coronary angioplasty & $114(0.12 \%)$ & $435(0.43 \%)$ & $1007(0.99 \%)$ & $<0.001$ \\
\hline Diagnostic cardiac catheterisation & $1830(1.85 \%)$ & $4804(4.77 \%)$ & $8408(8.29 \%)$ & $<0.001$ \\
\hline Insertion; revision; replacement; removal of cardiac pacemaker or cardioverter defibrillator & $1228(1.24 \%)$ & $4277(4.24 \%)$ & $8146(8.04 \%)$ & $<0.001$ \\
\hline
\end{tabular}

\section{Other predictors of mortality}

We did not find an increased risk of in-hospital mortality in patients with HF of black race or of Hispanic ethnicity. Previous studies in HF have revealed contrasting findings in regard to the effect of race in HF. Studies have found higher mortality, ${ }^{19}$ lower mortality ${ }^{20}$ and also no difference in mortality ${ }^{21}$ between blacks and other races. Also, another reason could be that the socioeconomic factors that usually contribute to worse outcomes in the ethnic minorities, ${ }^{22}$ may not affect 'in-hospital' outcomes as all patients have equal access to care while being in the hospital. A recent study involving the US veterans found that black patients with equal access to care have in fact lower all-cause mortality. ${ }^{23}$

\section{Limitations}

This was a study with a large sample size, equal representation of males and females and results were consistent in various subgroup analyses. However, there are some important limitations. First, we were unable to analyse outcomes based on the aetiology of cardiomyopathy (ischaemic vs non-ischaemic) and did not have information on the exact cause of death in the hospital (cardiac vs non-cardiac). While, 30-day mortality would have been a more objective measure of the quality of care being delivered across different centre volume groups, we did not have information on the outcome of death following discharge from the hospital and thus could not include 30-day mortality in our outcomes analysis. However, similar methodologies have been used in previous studies evaluating the effect of 'in-hospital' characteristics on 'in-hospital mortality. ${ }^{24}$ Second, although, there has been some criticism about the use of APR-DRGs for measuring severity of illness and risk for mortality to determine case-mix index; APR-DRGs still have high clinical validity ${ }^{12} 1325$ and are used by payers for risk-adjusted payments to the hospitals and by hospitals for public quality reporting. Third, we could not evaluate the effect of other hospital characteristics on outcomes like teaching versus non-teaching or location of the hospital (urban vs rural), level of physician training, physician volume, presence of a HF programme and/or dedicated HF nursing staff. Fourth, since the unit of analysis for this study was a HFH, same patients may have been hospitalised more than once over the study period. Finally, we did not have information on other factors that may contribute to outcomes like information on goals of care, do-not-resuscitate (DNR) status or comfort care measures. Hence, we are unable to delineate the exact reasons behind this observed phenomenon. Future studies are required to answer this.

\section{CONCLUSIONS}

Centre volume is an important determinant of in-hospital mortality in patients with HF. Higher centre volume is associated with lower HFH mortality but increased HFH costs and increased cardiac procedures in a cohort that included both Medicare and non-Medicare beneficiaries.

\section{Main messages}

- Higher centre volume was associated with a lower in-hospital mortality in heart failure hospitalisations (HFH) in a large cohort of Medicare and non-Medicare patients.

- While high centre volume group hospitals had lower HFH mortality, they were also associated with increased HFH costs and increased use of cardiac procedures.

- For the sickest group of patients with HF, the effect of centre volume on HFH mortality was modest but the increase in costs was significant.

\section{Current research questions}

- We were unable to identify the exact reason for the observed reduction in HFH mortality with increased centre volume. Future research is needed to answer this.

- Future research should also address whether special heart failure (HF) programmes with dedicated $\mathrm{HF}$ nursing or $\mathrm{HF}$ units in the hospital have any major role in lowering $\mathrm{HFH}$ mortality.

- Finally, we need to identify patient characteristics who would benefit the most by an early triage to higher centre volume hospitals for improving HFH outcomes but keeping the HFH costs in control.

Contributors SM and UPJ designed the study, wrote the first draft of the manuscript and had full access to the data. SM did the statistical analysis. DS, OS, SRP and JJS were responsible for critical review and changes in the manuscript.

Competing interests None declared.

Provenance and peer review Not commissioned; externally peer reviewed.

\section{REFERENCES}

1 Hunt SA, Abraham WT, Chin MH, et al. 2009 Focused update incorporated into the ACC/AHA 2005 guidelines for the diagnosis and management of heart failure in adults: a report of the American College of Cardiology Foundation/American Heart Association Task Force on Practice Guidelines developed in collaboration with the International Society for Heart and Lung Transplantation. J Am Coll Cardiol 2009;53:e1-90

2 Heidenreich PA, Trogdon JG, Khavjou OA, et al. Forecasting the future of cardiovascular disease in the United States: a policy statement from the American heart association. Circulation 2011;123:933-44.

3 Gheorghiade M, Vaduganathan M, Fonarow GC, et al. Rehospitalization for heart failure: problems and perspectives. J Am Coll Cardiol 2013;61:391-403.

4 Chen J, Normand S-LT, Wang Y, et al. National and regional trends in heart failure hospitalization and mortality rates for Medicare beneficiaries, 1998-2008. JAMA 2011;306:1669-78.

5 McMurray JJ, Adamopoulos S, Anker SD, et al. ESC Guidelines for the diagnosis and treatment of acute and chronic heart failure 2012: The Task Force for the 
Diagnosis and Treatment of Acute and Chronic Heart Failure 2012 of the European Society of Cardiology. Developed in collaboration with the Heart Failure Association (HFA) of the ESC. Eur J Heart Fail 2012;14:803-69.

6 Russo MJ, Iribarne A, Easterwood R, et al. Post-heart transplant survival is inferior at low-volume centers across all risk strata. Circulation 2010;122(Suppl 1):S85-91.

7 Post PN, Kuijpers M, Ebels T, et al. The relation between volume and outcome of coronary interventions: a systematic review and meta-analysis. Eur Heart $J$ 2010;31:1985-92.

8 Light TD, Latenser BA, Kealey GP, et al. The effect of burn center and burn center volume on the mortality of burned adults - an analysis of the data in The National burn repository. J Burn Care Res 2009;30:776-82.

9 Nathens $A B$, Jurkovich GJ, Maier RV, et al. Relationship between trauma center volume and outcomes. JAMA 2001;285:1164-71.

10 Handford CD, Rackal JM, Tynan A-M, et al. The association of hospital, clinic and provider volume with HIV/AIDS care and mortality: systematic review and meta-analysis. AIDS Care 2012;24:267-82.

11 Giri S, Pathak R, Aryal MR, et al. Impact of hospital volume on outcomes of patients undergoing chemotherapy for acute myeloid leukemia: a matched cohort study. Blood 2015;125:3359-60.

12 Reed SD, Cramer SC, Blough DK, et al. Treatment with tissue plasminogen activator and inpatient mortality rates for patients with ischemic stroke treated in community hospitals. Stroke 2001;32:1832-40.

13 Kollef MH, Hamilton CW, Ernst FR. Economic impact of ventilator-associated pneumonia in a large matched cohort. Infection Control 2012;33:250-6.

14 Joynt KE, Orav EJ, Jha AK. The association between hospital volume and processes, outcomes, and costs of care for congestive heart failure. Ann Intern Med 2011;154:94-102.
15 Ghaferi AA, Birkmeyer JD, Dimick JB. Variation in hospital mortality associated with inpatient surgery. N Engl J Med 2009;361:1368-75.

16 Mosterd A, Cost B, Hoes AW, et al. The prognosis of heart failure in the general population. Eur Heart J 2001;22:1318-27.

17 Oren RM. Hyponatremia in congestive heart failure. Am J Cardiol 2005;95:2-7.

18 Allen LA, Felker GM, Pocock $\mathrm{S}$, et al. Liver function abnormalities and outcome in patients with chronic heart failure: data from the Candesartan in Heart Failure: Assessment of Reduction in Mortality and Morbidity (CHARM) program. Eur J Heart Fail 2009;11:170-7.

19 Dries DL, Strong MH, Cooper RS, et al. Efficacy of angiotensin-converting enzyme inhibition in reducing progression from asymptomatic left ventricular dysfunction to symptomatic heart failure in black and White patients. J Am Coll Cardiol 2002:40:311-17.

20 Rathore SS, Foody JM, Wang Y, et al. Race, quality of care, and outcomes of elderly patients hospitalized with heart failure. JAMA 2003;289:2517-24.

21 Mathew J, Wittes J, McSherry F, et al. Racial differences in outcome and treatment effect in congestive heart failure. Am Heart J 2005;150:968-76.

22 Singh GK, Azuine RE, Siahpush M, et al. All-cause and cause-specific mortality among US youth: socioeconomic and rural-urban disparities and international patterns. J Urban Health 2013;90:388-405.

23 Kovesdy $C P$, Norris $K C$, Boulware $L E$, et al. Association of race with mortality and cardiovascular events in a large cohort of US veterans. Circulation 2015;132:1538-48.

24 Needleman J, Buerhaus P, Pankratz VS, et al. Nurse staffing and inpatient hospital mortality. N Engl J Med 2011;364:1037-45.

25 lezzoni LI, Ash AS, Shwartz M, et al. Predicting in-hospital deaths from coronary artery bypass graft surgery: do different severity measures give different predictions? Med Care 1998;36:28-39. 